\title{
Search for satellites near comet 67P/Churyumov-Gerasimenko using Rosetta/OSIRIS images
}

\author{
I. Bertini $^{1}$, P. J. Gutiérrez ${ }^{2}$, L. M. Lara ${ }^{2}$, F. Marzari ${ }^{3}$, F. Moreno ${ }^{2}$, M. Pajola ${ }^{1}$, F. La Forgia ${ }^{3}$, H. Sierks ${ }^{4}$, C. Barbieri $^{3}$, \\ P. Lamy $^{5}$, R. Rodrigo ${ }^{6,7}$, D. Koschny ${ }^{8}$, H. Rickman ${ }^{9,10}$, H. U. Keller ${ }^{11}$, J. Agarwal ${ }^{4}$, M. F. A'Hearn ${ }^{12}$, M. A. Barucci ${ }^{13}$, \\ J.-L. Bertaux ${ }^{14}$, G. Cremonese ${ }^{15}$, V. Da Deppo ${ }^{16}$, B. Davidsson ${ }^{9}$, S. Debei ${ }^{17}$, M. De Cecco ${ }^{18}$, F. Ferri ${ }^{1}$, S. Fornasier ${ }^{13,19}$, \\ M. Fulle ${ }^{20}$, L. Giacomini ${ }^{21}$, O. Groussin ${ }^{22}$, C. Güttler ${ }^{4}$, S. F. Hviid ${ }^{23}$, W.-H. Ip ${ }^{24,25}$, L. Jorda ${ }^{22}$, J. Knollenberg ${ }^{23}$, \\ J. R. Kramm ${ }^{4}$, E. Kührt ${ }^{23}$, M. Küppers ${ }^{26}$, M. Lazzarin ${ }^{3}$, J. J. Lopez Moreno ${ }^{2}$, S. Magrin ${ }^{3}$, M. Massironi ${ }^{21}$, \\ H. Michalik ${ }^{27}$, S. Mottola ${ }^{23}$, G. Naletto ${ }^{28,16,1}$, N. Oklay ${ }^{4}$, N. Thomas ${ }^{29}$, C. Tubiana ${ }^{4}$, and J.-B. Vincent ${ }^{4}$ \\ (Affiliations can be found after the references)
}

Received 27 February 2015 / Accepted 11 May 2015

\begin{abstract}
Context. The European Space Agency Rosetta mission reached and started escorting its main target, the Jupiter-family comet 67P/ChuryumovGerasimenko, at the beginning of August 2014. Within the context of solar system small bodies, satellite searches from approaching spacecraft were extensively used in the past to study the nature of the visited bodies and their collisional environment.

Aims. During the approaching phase to the comet in July 2014, the OSIRIS instrument onboard Rosetta performed a campaign aimed at detecting objects in the vicinity of the comet nucleus and at measuring these objects' possible bound orbits. In addition to the scientific purpose, the search also focused on spacecraft security to avoid hazardous material in the comet's environment.

Methods. Images in the red spectral domain were acquired with the OSIRIS Narrow Angle Camera, when the spacecraft was at a distance between $5785 \mathrm{~km}$ and $5463 \mathrm{~km}$ to the comet, following an observational strategy tailored to maximize the scientific outcome. From the acquired images, sources were extracted and displayed to search for plausible displacements of all sources from image to image. After stars were identified, the remaining sources were thoroughly analyzed. To place constraints on the expected displacements of a potential satellite, we performed Monte Carlo simulations on the apparent motion of potential satellites within the Hill sphere.

Results. We found no unambiguous detections of objects larger than $\sim 6 \mathrm{~m}$ within $\sim 20 \mathrm{~km}$ and larger than $\sim 1 \mathrm{~m}$ between $\sim 20 \mathrm{~km}$ and $\sim 110 \mathrm{~km}$ from the nucleus, using images with an exposure time of $0.14 \mathrm{~s}$ and $1.36 \mathrm{~s}$, respectively. Our conclusions are consistent with independent works on dust grains in the comet coma and on boulders counting on the nucleus surface. Moreover, our analysis shows that the comet outburst detected at the end of April 2014 was not strong enough to eject large objects and to place them into a stable orbit around the nucleus. Our findings underline that it is highly unlikely that large objects survive for a long time around cometary nuclei.
\end{abstract}

Key words. comets: general - comets: individual: 67P/Churyumov-Gerasimenko - planets and satellites: detection - techniques: photometric

\section{Introduction}

The detection and study of small-body satellites is an important tool for investigating the nature, origin, and evolution of asteroids and comets. Measuring the orbit of small companions allows determining the mass of the system and of the primary. From this, its bulk density is derived when the volume is known. This provides hints on the physical composition of the object and its internal structure. Studying the connected systems also provides clues on the collisional events that occurred during the early stages of the formation of the solar system and its subsequent evolution (Merline et al. 2002).

At the time of writing (beginning of May 2015), we know of 256 small bodies that have companions of different sizes ${ }^{1}$. Among them there are 55 near-Earth asteroids (NEAs), 20 Marscrossers, 97 main belt asteroids (MBAs), 4 Jupiter Trojans, and 80 trans-Neptunian objects (TNOs). Three TNOs that display complex systems belong to the Centaur class, which are assumed

\footnotetext{
1 http://www.johnstonsarchive.net/astro/asteroidmoons. html
}

to be composed of objects with an orbit intermediate between TNOs and short-period comets (Levison \& Duncan 1997).

Spacecraft encounters allow satellite searches and discoveries down to sizes much smaller than possible from Earth, which also adds the advantage of effectively investigating the space closer to the objects. Several satellite searches were performed using data from NASA, JAXA, and ESA missions. We mention the studies of NASA/Galileo at (951) Gaspra (Belton et al. 1992) and (243) Ida (Belton et al. 1995), NASA/NEAR at (253) Mathilde (Veverka et al. 1999) and (433) Eros (Veverka et al. 2000), JAXA/Hayabusa at (25143) Itokawa (Fuse et al. 2008), ESA/Rosetta at (21) Lutetia (Bertini et al. 2012), and finally NASA/Dawn at (4) Vesta (Memarsadeghi et al. 2013). Except for the encounter with Ida, which provided the first direct and definitive evidence of the existence of asteroid companions with the serendipitous discovery of the small moon Dactyl in 1993, all other searches were unsuccessful in detecting small companions. These studies allowed placing important constraints on the size limit of possible satellites, however, providing hints on the collisional history of the primary bodies.

A double nucleus with two possibly bound components was claimed to explain the photometric anisotropies in the inner 
coma of the large comet C/1995 O1 Hale-Bopp based on both ground-based (Marchis et al. 1999) and HST (Sekanina 1997) data. However, as underlined in Noll et al. (2006), Weaver \& Lamy (1997) reported no evidence of the second companion using the same HST dataset, showing that no final univocal conclusion on the binary nature of the system could be derived. Decimeter-sized icy particles were found in the close vicinity of the nucleus of the hyperactive comet 103P/Hartley 2 during the flyby of the NASA/EPOXI spacecraft performed on 2010 November 4 (A'Hearn et al. 2011; Kelley et al. 2013; Hermalyn et al. 2013). Moreover, several cometary nuclei visited by space missions (e.g., 1P/Halley, 19P/Borrelly, 103P/Hartley 2, and $67 \mathrm{P} /$ Churyumov-Gerasimenko itself) showed complex irregular shapes that can be interpreted as the results of the evolution of contact binary systems. Despite these interesting considerations, no classical satellite searches have been performed for comets, as was extensively done for asteroids, and no solid material larger than $\sim 1 \mathrm{~m}$ orbiting a comet has ever been unambiguously discovered.

During the approach to comet 67P/ChuryumovGerasimenko in July 2014, before the orbit insertion performed at the beginning of August 2014, the two-camera instrument OSIRIS (Keller et al. 2007) onboard Rosetta took several images with the purpose of detecting and studying possible objects orbiting the comet in the vicinity of the nucleus. The search had the additional aim to ensure spacecraft safety. The discovery of solid blocks close to the nucleus would have implied that special care was necessary so that the spacecraft trajectory would not cross any orbiting material. An appropriate observational strategy was defined so that the data acquisition and reduction processes were optimized, maximizing the possibility of detecting and measuring the orbital arc of a possible small companion. We here present the adopted observational strategy and the data analysis, together with the results of our investigation.

\section{Observational strategy}

It is well known that satellite searches from spacecraft images are affected by several problems such as the fast motion of the camera with respect to the target and the bona-fide detection of interesting point-like objects against background stars, cosmicray events, and CCD defects (Merline et al. 2002).

One OSIRIS image series was specifically devoted to the search for potential satellites during the comet approach phase on July 20, 2014, using the high-resolution Narrow Angle Camera (NAC) telescope. The images were taken when the comet was at 3.69 AU from the Sun. The distance to the comet decreased from $5785 \mathrm{~km}$ to $5463 \mathrm{~km}$ from the beginning to the end of the series. The phase angle of the observations was $7^{\circ}$. The series consisted of 18 short- and 18 long-exposure images divided into three consecutive frames so as to reduce the contamination from cosmic-ray hits through their lack of persistence, for a total of six different short- and long-exposure runs. Each run was separated by $1 \mathrm{~h}$ except for the last one, which was taken $7 \mathrm{~h}$ apart. The satellite search series therefore covered almost an entire comet rotation period. Within each run, the time separation between consecutive frames corresponded to $20 \mathrm{~s}$ except for the 5th run, where it was $10 \mathrm{~s}$. Within the series, the same short- $(0.14 \mathrm{~s})$ or long- $(1.36 \mathrm{~s})$ exposure time was used to reach the same limiting magnitude and avoid difficulties when looking for correspondences among the three frames. The long-exposure times were selected with the aim of avoiding stellar background smearing, which could have complicated the star identification.
Short-exposure frames were taken for their relevance if a large satellite had been detected, since they would have provided unsaturated views of the object. The NAC broadband orange filter (with a central wavelength and FWHM of $649.2 \mathrm{~nm}$ and $84.5 \mathrm{~nm}$, respectively) in the visible red domain was chosen to provide the best $\mathrm{S} / \mathrm{N}$ for possible satellites within a fixed exposure time. Considering the image scale, the observed field of view (FoV) covered the inner $\sim 110 \mathrm{~km}$ from the comet optocenter. When we mention a distance, it refers to the "projected distance". Assuming a Hill sphere radius of $\sim 650 \mathrm{~km}$ derived from the measurement of the comet mass by the Rosetta Radio Science Investigation (RSI) instrument, namely $1.0 \times 10^{13} \mathrm{~kg}$ (Sierks et al. 2015, and references therein), and from the heliocentric distance-dependent formula in Hamilton \& Burns (1991), we note that our FoV intersected a three-dimensional space corresponding to $\sim 37 \%$ of the comet gravitational sphere of influence.

The first and the last images obtained for the satellite search are shown in Fig. 1.

\section{Analyzing the data}

When we analyzed the data, we first estimated the expected results for a potential satellite by performing a Monte Carlo simulation. To do this, we selected 50000 clones randomly located inside the Hill sphere, with a random velocity with a smaller modulus than the escape velocity. We calculated the clone positions within the CCD sensor reference frame for the time in which the long-exposure images were taken. A value of $1.0 \mathrm{~m} \mathrm{~s}^{-1}$ was used for the escape velocity, in accordance with Sierks et al. (2015). In a first approach, acceleration effects on the potential satellite were neglected. The spacecraft position and frame orientations were derived using appropriate spice kernels.

After calculating the clone positions, we measured their displacements for three different cases: (1) within a single exposure time to determine whether the potential satellite showed a track in a single frame; (2) between individual images to determine whether it was possible to apply median averaging of images within the same run in order to eliminate possible cosmic rays and spurious signals; (3) between runs of images to limit the radius search for displacements of the potential satellite.

With this analysis we found that clones did not move more than 0.5 px within each run (see Fig. 2), allowing us to median combine the three frames within each run to effectively eliminate cosmic-ray effects. Moreover, the theoretical displacement from run to run was calculated to be smaller than $50 \mathrm{px}$ within the first five runs, separated by $1 \mathrm{~h}$. The displacement of the potential satellite between runs 5 and 6 may have been practically any value, depending on the clone velocity, because the time separation between these two runs was $7 \mathrm{~h}$ (see Fig. 3). For this reason, we focused on the analysis of the first five runs and kept the sixth run in reserve in case we detected a potential satellite within the first five runs. To detect potential satellites, we started working with only the median average of the three long-exposure images of each run.

We then defined the limiting flux for the object detection. OSIRIS frames are photometrically calibrated using the frequently tested instrument calibration pipeline described in Tubiana et al. (2015a). First, we used the SExtractor code (Bertin $\&$ Arnouts 1996) with a proper set-up for detecting light sources with a flux $\geq 3 \sigma$, where $\sigma$ is the background level, within an aperture radius 2 px larger than the filter point spread function (PSF) full width at half maximum (FWHM) that was estimated 


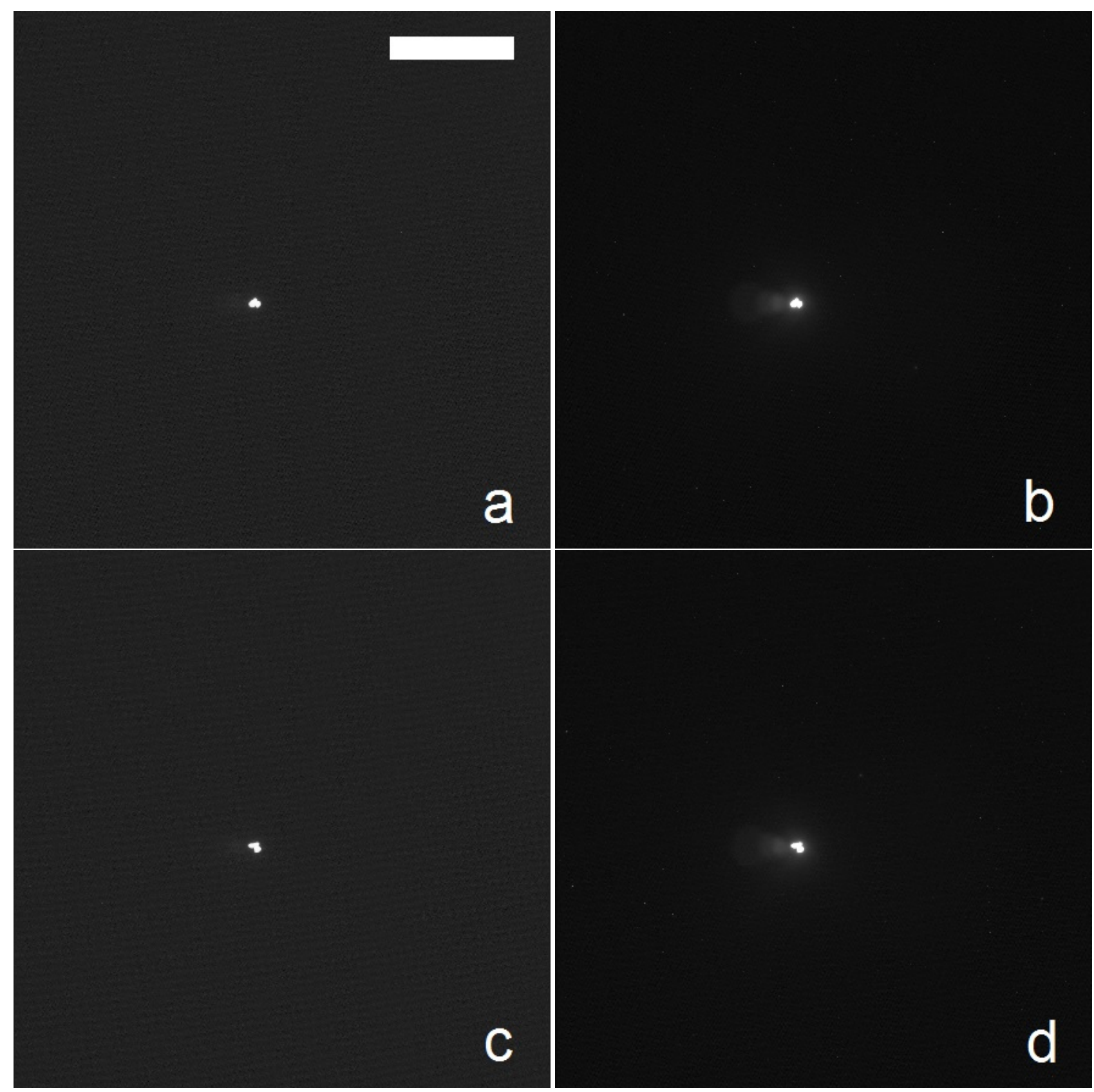

Fig. 1. First and last runs short (a), c)) and long exposure (b), d)) median-combined frames taken for the satellite search on July 20, 2014. The horizontal white bar in a) corresponds to a scale length of $50 \mathrm{~km}$ at the comet distance.

from calibrations to be $\sim 1.8$ px (Magrin et al. 2015). To ensure full control of the source detections, we performed manual photometry and estimated the flux in a circular aperture of 2 px radius of all the sources detected by SExtractor. We also estimated the local sky background. We only considered sources with a flux three times the standard deviation of all sky values for the subsequent study. This was defined as the source threshold. The highest source threshold value of the different runs was set as our detection limit (see Table 1). This resulted in considering light sources with fluxes in the 2 px aperture larger than $8.9 \times 10^{-8}\left[\mathrm{~W} \mathrm{~m}^{-2} \mathrm{~nm}^{-1} \mathrm{sr}^{-1}\right]$. We correlate the fluxes with the $R$ and $V$ magnitudes of the nucleus below.

The stellar background was then identified by correlating the different median-averaged images through small shifts and rotations to cause the brightest 100 sources found in the images with SExtractor to overlap. After correlating the frames, the stars were identified as the sources located in the same pixel, with a tolerance lower than $5 \mathrm{px}$. The value of this error depends on the geometric distortion correction goodness of the field, on the central pixel identification error of the sources, and on the correlation algorithm itself.

The remaining sources, found to be $\sim 200$ for each run (see Table 1), might in principle be spurious signals, undetected stars, CCD defects, and, of course, potential satellites. These remaining sources, shown in Fig. 4, were considered for further investigation. The plot shows the sources that were not identified as stars in the different runs displayed in the same frame, obtained after correlation. Clouds of sources around the central position of the CCD are due to the edge of the window that we were forced to define to avoid the ghost of the nucleus, which is always present in our images and cannot be considered reliable. This resulted in cutting out a square of 400 px size centered on 


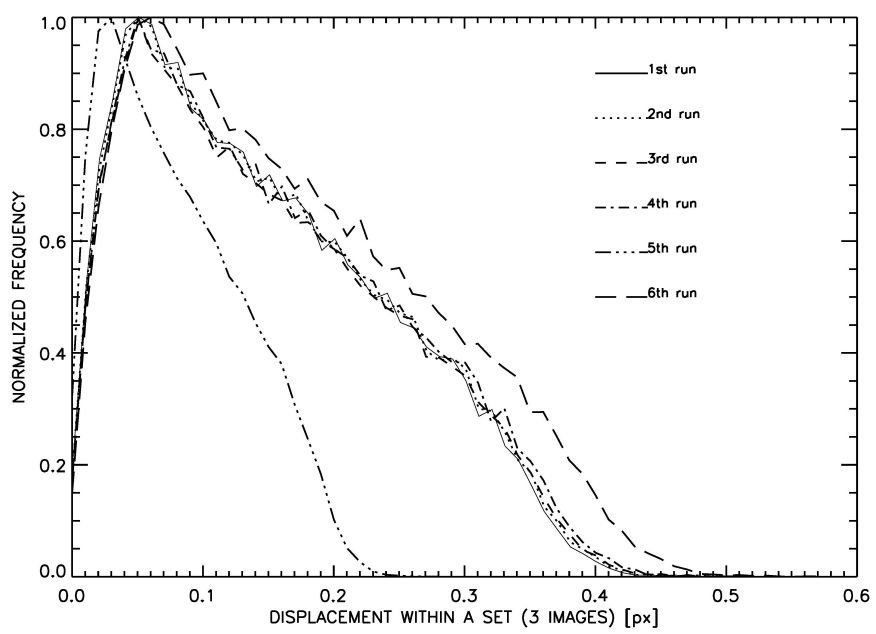

Fig. 2. Normalized frequency of satellite clone displacements within a single run composed of three consecutive images. Results are shown for all six runs covering the satellite search.

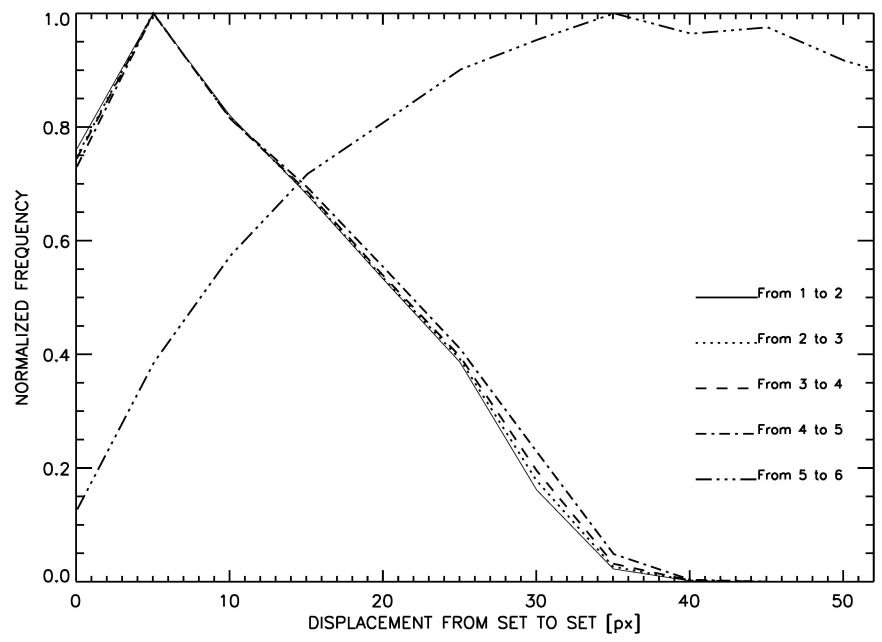

Fig. 3. Normalized frequency of satellite clone displacements between two consecutive runs. Results are shown for displacements between runs 1 and 2 (continuous line), runs 2 and 3 (dotted line), runs 3 and 4 (dashed line), runs 4 and 5 (dashed-dotted line), and runs 5 and 6 (dashed-triple-dotted line). The last curve depicts the large displacement that was the reason for discarding run 6 from the satellite analysis.

Table 1. Image series dedicated to the satellite search.

\begin{tabular}{cccc}
\hline \hline Image & $3 \sigma\left[\mathrm{W} \mathrm{m}^{-2} \mathrm{~nm}^{-1} \mathrm{sr}^{-1}\right]$ & Sources & Non-stars \\
\hline RUN1 & $8.3 \times 10^{-8}$ & 1316 & 200 \\
RUN2 & $6.4 \times 10^{-8}$ & 1398 & 221 \\
RUN3 & $7.2 \times 10^{-8}$ & 1260 & 151 \\
RUN4 & $8.9 \times 10^{-8}$ & 1273 & 185 \\
RUN5 & $8.1 \times 10^{-8}$ & 1451 & 224 \\
\hline
\end{tabular}

Notes. Sources and non-stars are the total number of detected sources with a flux higher than $3 \sigma$ and the number of detected sources that are not identified as stars.

the comet nucleus, corresponding to the first $\sim 20 \mathrm{~km}$ around the comet optocenter and to the inner $\sim 4 \%$ of the Hill sphere. In the displayed frame, a satellite would appear as an apparently moving object showing a track corresponding to its position at the different runs (e.g., points A and B in Fig. 4). These tracks

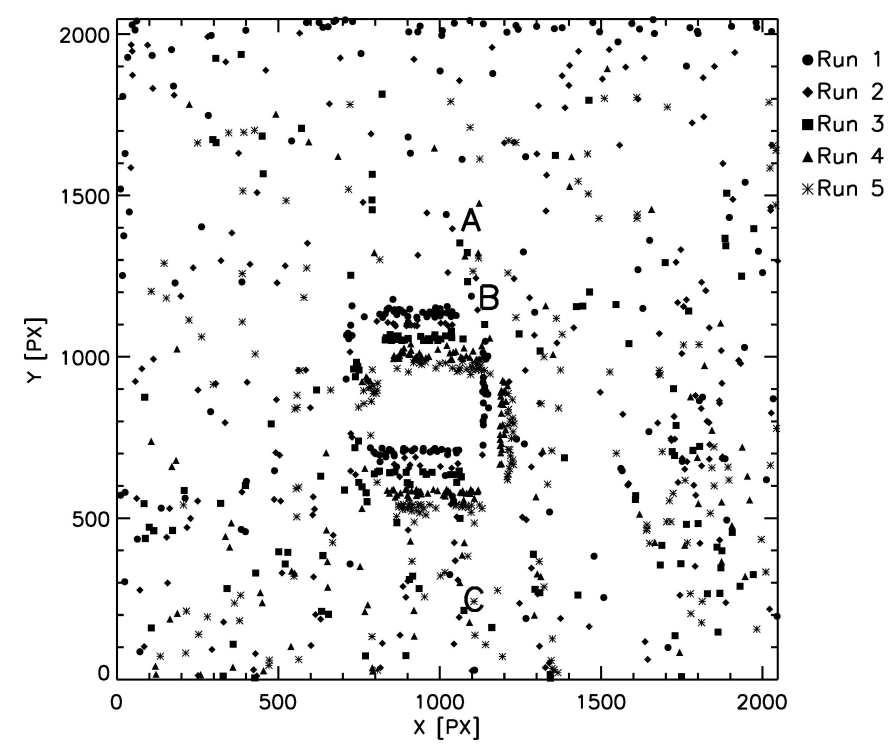

Fig. 4. Correlated long-exposure image showing all detected light sources not identified as stars. A, B, and C display a track but were discarded as potential satellites after the analysis.

were verified to be CCD defects, which also appear in images that are unrelated to the satellite search series and correspond to the same $(x, y)$ position when displayed in the original uncorrelated frames. The main problem of identifying a satellite close to the detection limit is that the potential satellite is not necessarily present in all five considered runs. The satellite can remain undetected in some of the runs, for example, because of intrinsic rotational variability or high background level. To find and detect possible tracks of potential satellites, we therefore considered the information obtained from the clone simulations. We only took into account for a thorough analysis the sources showing a potential track that appeared in at least three runs out of the five. With the Monte Carlo analysis we verified that all the potential satellites appeared to move in a straight line from top to bottom in the CCD frame (see Fig. 5) and that the displacement from run to run was exactly the same, within at most $2 \mathrm{px}$. This "error" would also include the source pixel identification, bearing in mind that the PSF FWHM is $\sim 1.8 \mathrm{px}$. As the considered space region is beyond $20 \mathrm{~km}$ from the nucleus center, gravitational accelerations were not considered in the simulations. We assumed that they may be a second-order effect, on the order of the pixel scale at most. The gravitational acceleration is probably lower than $10^{-5} \mathrm{~m} \mathrm{~s}^{-2}$ for cometocentric distances larger than $6 \mathrm{~km}$ from the surface, given the mass of 67P. This acceleration, in modulus, would produce a displacement on the order of or smaller than the pixel size scale from run to run (except for runs 5 to 6 , where the acceleration might have a noticeable effect) and, in any case, smaller than the PSF FWHM. In the search for source tracks, we additionally imposed from the clone study that the separation from run to run of the potential satellite had to be of the same order, with a safe margin of $3 \mathrm{px}$, and that it could not be larger than 50 px. From our analysis, we found four sources showing a compatible theoretical track, but they were either hot pixels or CCD defects, as confirmed in unrelated images (as the tracks identified by A and B in Fig. 4). After a thorough search, the most likely track for a candidate satellite was the track identified by the letter $\mathrm{C}$ in Fig. 4. This track consisted of three sources appearing in runs 3,4 , and 5 , as indicated in Table 2. This potential common source appearing in at least three 
Table 2. Candidate satellite data.

\begin{tabular}{cccccc}
\hline \hline Image & Position1 & Distance1 [px] & Position2 & Distance2 [px] & Flux [W m ${ }^{-2} \mathrm{~nm}^{-1} \mathrm{sr}^{-1}$ ] \\
\hline RUN3 & $(1039,303)$ & & $(1075,214)$ & & $2.3 \times 10^{-6}$ \\
RUN4 & $(1043,308)$ & 7 & $(1091,177)$ & 41 & $4.5 \times 10^{-7}$ \\
RUN5 & $(1039,315)$ & 8 & $(1109,137)$ & 43 & $1.7 \times 10^{-6}$ \\
\hline
\end{tabular}

Notes. Positions 1 and 2 are the CCD $(x, y)$ coordinates of the candidate satellite in the original and correlated frames. Similarly, distance 1 and 2 are the separations of the sources in the different runs in the original and correlated frames. The keyword flux indicates the photometric flux of the object.

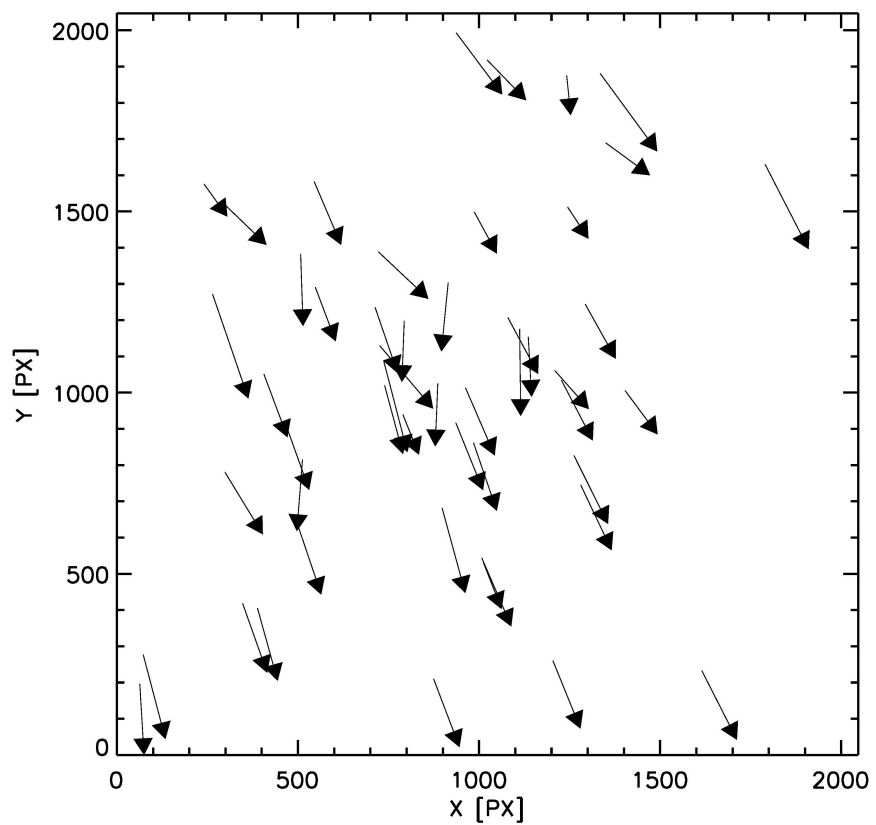

Fig. 5. Apparent motion of 50 satellite clones in the correlated frame from the first image of run 1 to the last image of run 5. The arrow orientation indicates the direction of the apparent motion in the CCD frame taking into account the spacecraft position and pointing as included in the spice kernels and a random velocity lower than the escape velocity. The arrow length provides an indication of the total displacement on the CCD frame due to the satellite clone motion.

runs would have had a separation from run to run that was very close to the maximum of the expected separation from the clone study (see Fig. 3), and the difference in distance from runs 3-4 to runs 4-5 was smaller than 2 pix. The related photometric flux showed a variation of one order of magnitude, which might be due to a very elongated shape (it would correspond to an axis ratio of $\sim 3$ ). All these circumstances defined this potential source as a possible candidate. Nevertheless, this track showed a peculiarity observable when the $(x, y)$ coordinates in the original CCD images were considered. The potential source was characterized by a small swaying movement in the $(x)$ coordinate. This movement had a significant amplitude of 4 pixels, larger than the PSF FWHM. The clone study was used to detect possible satellites with such an apparent motion, and we found no single case characterized by this apparent displacement. Additionally, if the change from run 3 to run 4 is considered, the expected position at run 5 should have been $(1047,313)$ in the original CCD image, that is, more than five times the PSF FWHM in the $(x)$ coordinate. These arguments led us to conclude that the apparent track was just a coincidence and did not correspond to a real source detected in three out of the five runs.

Based on our thorough analysis of the remaining sources and imposing the described constraints, we therefore conclude that we found no unambiguous detections of objects in the $\sim[20-110] \mathrm{km}$ range from the nucleus up to a limiting flux of $8.9 \times 10^{-8}\left[\mathrm{~W} \mathrm{~m}^{-2} \mathrm{~nm}^{-1} \mathrm{sr}^{-1}\right]$.

\subsection{Space close to the nucleus}

To analyze the space close to the nucleus, which was cut off in the long-exposure images because of the bright ghost of the nucleus, we took into account the short-exposure frames belonging to the same series.

We applied the same analysis as performed on the longexposure images, this time considering the entire frame, including the space close to the nucleus and the nucleus itself. Since we aimed to study the region close to the nucleus, we performed additional simulations with clones that had a Keplerian acceleration according to their distance from the nucleus. Even though acceleration may be comparatively large close to the nucleus, its effect was not noticeable in the images we considered since it was found to be smaller than $1 \mathrm{px}$ in all images given the short exposure time. Based on our simulations, we therefore conclude that the constraints imposed from the long-exposure images still hold.

The main problem here was defining a proper detection threshold because the electronic noise resulted in background patterns that hindered defining a limiting $\mathrm{S} / \mathrm{N}$.

We therefore relaxed the SExtractor detection limit. With a trial-and-error procedure, we defined the signal of $4.5 \sigma$ ( $\sigma$ is always the background level) as the lowest value that produced reliable detections. This resulted in considering light sources with a flux higher than $3.0 \times 10^{-6}\left[\mathrm{~W} \mathrm{~m}^{-2} \mathrm{~nm}^{-1} \mathrm{sr}^{-1}\right]$.

This search yielded the correlated image shown in Fig. 6. In that figure, several apparent moving objects can be clearly identified. Four of them, as those labeled A and B, correspond to the aforementioned CCD defects. The object labeled $\mathrm{E}$ is the comet nucleus, and D is a spurious detection due to the nucleus ghost.

We therefore finally conclude that we found no unambiguous detections of objects within $\sim 20 \mathrm{~km}$ from the nucleus up to a limiting flux of $3.0 \times 10^{-6}\left[\mathrm{~W} \mathrm{~m}^{-2} \mathrm{~nm}^{-1} \mathrm{sr}^{-1}\right]$.

\section{Estimating the limiting size}

Since our search for objects in the vicinity of the comet produced a negative result, we determined the limiting size for any object that might be present, but remain undetectable in our images.

\subsection{Measuring the limiting magnitude}

To determine the limiting size of possible solid blocks for detection we first measured the limiting magnitude reached in our images both within $\sim 20 \mathrm{~km}$ and from $\sim 20 \mathrm{~km}$ up to $\sim 110 \mathrm{~km}$ from the comet optocenter. 


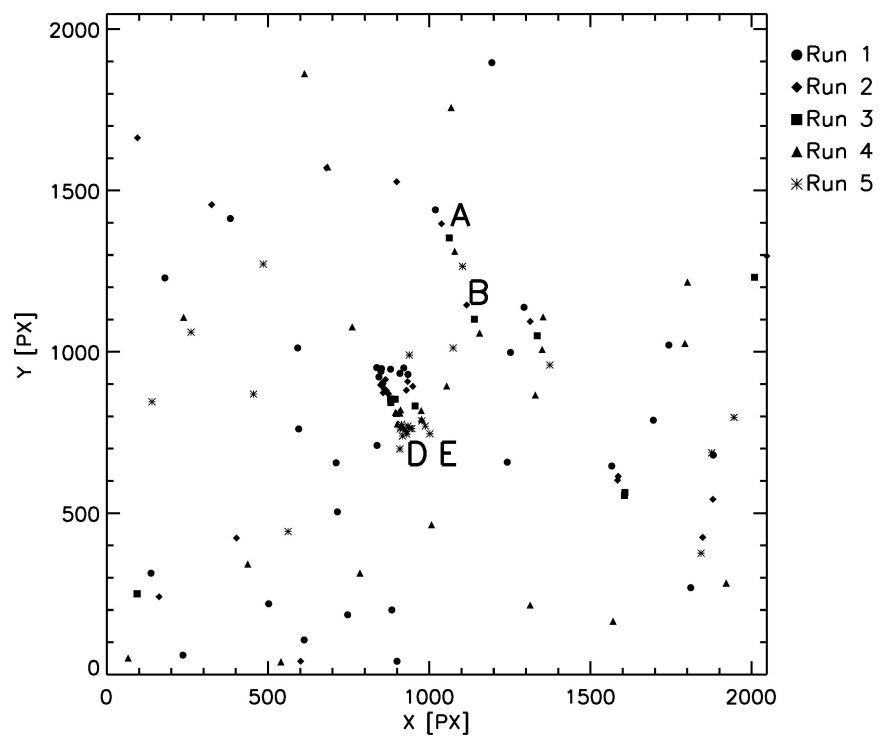

Fig. 6. Correlated short-exposure image showing all detected sources that are not identified as stars. A and B display a track but were discarded as potential satellites since they were identified as CCD defects. Objects labeled $\mathrm{E}$ and $\mathrm{D}$ are the comet nucleus and a spurious detection due to the nucleus ghost.

First, we converted the measured NAC broadband orange filter limiting fluxes into Kron-Cousins $R$ magnitudes. This was performed using the OSIRIS standard calibration fields, as in Mottola et al. (2014). Our limiting fluxes of $8.9 \times 10^{-8}$ and $3.0 \times 10^{-6}\left[\mathrm{~W} \mathrm{~m} \mathrm{~nm}^{-1} \mathrm{sr}^{-1}\right]$ corresponded to $R_{\lim }=14.63$ and $R_{\lim }=10.81$, respectively.

These are the smallest magnitudes that a satellite would have to have to be detected in the two different regions around the comet. Considering objects with the same photometric properties as the nucleus of 67P, the limiting magnitude in $R$ can be converted into a $V$-Johnson magnitude using the Johnson-KronCousins colors of the comet nucleus. Based on $(V-R)=0.54$ (Tubiana et al. 2011), $R_{\lim }=14.63$ and $R_{\lim }=10.81$ translated into $V_{\lim }=15.17$ and $V_{\lim }=11.35$, respectively.

\subsection{From limiting magnitude to limiting size}

After calculating $V_{\text {lim }}$, we were able to derive the absolute limiting magnitude, $H_{\mathrm{lim}}$. To do this, we used the photometrical models developed by Bowell et al. (1989). We used the hypothesis of a satellite that has the same photometric properties as the comet nucleus and considered the real geometry of observations to constrain the maximum distance and minimum phase angle of a possible satellite detected by the camera.

The input photometric slope-parameter, $G=-0.13 \pm 0.01$ was measured from OSIRIS unresolved images of the comet nucleus taken during the approaching phase (Fornasier et al. 2015).

To provide the observational geometry input needed to convert $V_{\text {lim }}$ into $H_{\text {lim }}$, we measured the three-dimensional positions of 100000 Monte Carlo virtual satellites filling up the entire Hill sphere. The absolute limiting magnitude was then converted into a diameter measurement using (Chesley et al. 2002):

$D[\mathrm{~km}]=\frac{1329 \times 10^{-0.2 H}}{\sqrt{p_{V}}}$,

where $p_{V}=0.061 \pm 0.001$ is the geometric $V$-band albedo of the comet nucleus (Fornasier et al. 2015).
Table 3. Final limiting sizes for potential satellites of 67P.

\begin{tabular}{cccc}
\hline \hline Image & $t_{\exp }[\mathrm{s}]$ & Size $[\mathrm{m}]$ & Error [m] \\
\hline RUN1 & 0.14 & 5.77 & 0.23 \\
& 1.36 & 1.00 & 0.11 \\
RUN2 & 0.14 & 5.73 & 0.24 \\
& 1.36 & 0.99 & 0.10 \\
RUN3 & 0.14 & 5.69 & 0.24 \\
& 1.36 & 0.98 & 0.10 \\
RUN4 & 0.14 & 5.66 & 0.23 \\
& 1.36 & 0.98 & 0.11 \\
RUN5 & 0.14 & 5.62 & 0.21 \\
& 1.36 & 0.97 & 0.10 \\
\hline
\end{tabular}

Notes. $t_{\exp }$ is the image exposure time within single runs. Size and error columns show our final results in measuring the limiting size of undetected objects and the associated error estimate.

Our final results are shown in Table 3 together with the associated error estimates. The largest contribution to the error on the size measurement comes from considering the variation of the observational geometry within the Hill sphere for the virtual satellites. The propagated error due to the uncertainty on the radiometric measurements coming from the OSIRIS calibration pipeline is negligible when compared to the effect of the variation of the observational geometry. For this reason, we report as final results the mean values of the size distribution found within the Hill sphere for every single run, and as associated error three times the stardard deviation of the size distribution. We conclude that $67 \mathrm{P}$ lacks objects larger than $\sim 6 \mathrm{~m}$ within the first $\sim 20 \mathrm{~km}$ from the comet nucleus and objects larger than $\sim 1 \mathrm{~m}$ at cometocentric distances between $\sim 20 \mathrm{~km}$ and $\sim 110 \mathrm{~km}$ at the time the observations were performed.

\section{Summary and discussion}

Images were taken with the aim to detect objects orbiting comet 67P/Churyumov-Gerasimenko on July 20, 2014, by the OSIRIS NAC telescope onboard the Rosetta mission during the approach to the comet at $\sim 5800 \mathrm{~km}$ distance both for scientific and spacecraft security reasons.

The negative outcome of our search led us to estimate a limiting size for possible undetected objects with the same photometric properties as the comet nucleus. We found no unambiguous detections of objects larger than $\sim 6 \mathrm{~m}$ at within the first $\sim 20 \mathrm{~km}$ from the nucleus and objects larger than $\sim 1 \mathrm{~m}$ at cometocentric distances between $\sim 20 \mathrm{~km}$ and $\sim 110 \mathrm{~km}$.

There are three most likely mechanisms that might produce a satellite for comet 67P: a subcatastrophic impact generating a cloud of fragments that re-accumulate into a satellite before reimpacting, comet splitting due to internal stresses caused either by activity (i.e., 73P/Schwassman-Wachmann 3), or tidal forces (i.e., comet Shoemaker-Levy 9), and radial gas drag forces lifting up boulders.

In the first case, the impact would have to have occurred when the comet was residing in the Kuiper Belt. Low-velocity cratering impacts $\left(\sim 1 \mathrm{~km} \mathrm{~s}^{-1}\right)$ within the belt may cause the ejection of a large number of small fragments into temporary orbits. The comet irregular shape and complex gravitational field may allow these fragments to survive for more than one period before falling back onto the comet, thus giving them enough time to collide with each other and accrete into a satellite. This mechanism would not be efficient if the comet originated from the Oort cloud. 
Cometary splitting is a common event: more than 40 split comets have been observed in the past $150 \mathrm{yr}$. The peak in the location of the breakup is close to perihelion at about $2 \mathrm{AU}$ from the Sun (Boehnhardt 2004). The relative speed of the fragments shortly after the fragmentation event usually appears to be high and not favorable to capture one or more fragments as satellites. However, it is possible that a whole spectrum of separation velocities is covered during the splitting event, and a particular combination of low-separation velocity, complex gravitational field, and outgassing force may inject a small component into a bound orbit.

Radial gas drag forces may lift meter-sized boulders from the nucleus surface, possibly injecting them into bound orbits, in particular if large anisotropies are present in both the gas drag and gravity force (Fulle 1997), as seems to be the case of comet 67P. Isolated boulders with a size of at most $\sim 6-7 \mathrm{~m}$ that are linked to possible gas activity ejection processes are seen in several areas on the nucleus surface (Pajola et al. 2015). After having been lifted up, several boulders might survive for one or more full orbital periods of the comet and could appear as small satellites. However, the sensitivity of our images allowed excluding objects with sizes larger than a few meters in late July 2014. This might be an indication that either the outgassing has not been strong enough to lift large boulders before that date or that the orbital survival of these objects through comet perihelion is difficult because of the strong perturbations from continuing outgassing. We also emphasize that our search only covered the inner $\sim 37 \%$ of the comet's Hill sphere calculated at the time of observations. Nevertheless, the images taken in July 2014 cover $\sim 87 \%$ of the Hill sphere calculated at perihelion $\left(r_{\text {Hill }}=215 \mathrm{~km}\right)$, where the efficiency in ejecting objects is highest. Our conclusions are therefore valid within the full extension of the comet's gravity field throughout its entire orbital period.

Our results are consistent with the findings in Rotundi et al. (2015), where a cloud of $\sim 350$ dust grains bound to the comet, at nucleocentric distances lower than $130 \mathrm{~km}$, was found in NAC orange filter images taken on August 4, 2014. The authors estimated these dust grains to have probably been placed in orbit just after the previous perihelion passage and to span from $4 \mathrm{~cm}$ to $\sim 2 \mathrm{~m}$ in size, being the last a crude upper limit obtained assuming that the brightest detected grains are also the farthest from the spacecraft. Taking into account the errors associated with the size measurements in these two independent works, our satellite search analysis confirms that objects larger than the upper limit in Rotundi et al. (2015) were not present in the vicinity of the nucleus. Similar considerations are valid when comparing our findings with the results of Davidsson et al. (2015), where the orbits of a few grains around the nucleus, with a size in the [0.14-0.50] m range, were calculated using OSIRIS Wide Angle Camera images taken on September 10, 2014, in the narrowband visible filter.

Moreover, OSIRIS detected a clear comet outburst between 2014 April 27 and 30, 2014, during the approaching phase. This impulsive event was estimated to have ejected a mass between $10^{3} \mathrm{~kg}$ and $10^{5} \mathrm{~kg}$ (Tubiana et al. 2015b). Our results indicate that the forces produced by the outburst were unable to lift up large chunks of material or that such blocks were unable to enter into orbit and remain close to the nucleus at the time of the satellite observations, three months after the impulsive event occurred.

Finally, considering all plausible formation scenarios, even if a satellite larger than a few meters was formed during the evolution of the comet, its survival would have been jeopardized by many adverse events. Close encounters with Jupiter, like the very deep one of 1959, may destabilize a satellite orbit through tidal forces and cause it to depart. In addition, changes in the pole direction due to strong outgassing close to perihelion cause significant variations in the gravity field (which is very irregular for 67P), and the satellite orbit may become unstable, leading to escape. Sublimation on a potentially small satellite would also strongly reduce its endurance, causing its fast erosion. The nongravitational forces related to the sublimation and the gas pressure released from the comet nucleus would also contribute to destabilizing its orbit.

In conclusion, even if there are different mechanisms that can cause the formation of a comet satellite, the adverse dynamical conditions that characterize the comet environment seem to play against its survival.

Acknowledgements. OSIRIS was built by a consortium of the Max-PlanckInstitut für Sonnensystemforschung, Göttingen, Germany, CISAS - University of Padova, Italy, the Laboratoire d'Astrophysique de Marseille, France, the Instituto de Astrofísica de Andalucia, CSIC, Granada, Spain, the Research and Scientific Support Department of the European Space Agency, Noordwijk, The Netherlands, the Instituto Nacional de Técnica Aeroespacial, Madrid, Spain, the Universidad Politéchnica de Madrid, Spain, the Department of Physics and Astronomy of Uppsala University, Sweden, and the Institut für Datentechnik und Kommunikationsnetze der Technischen Universität Braunschweig, Germany. The support of the national funding agencies of Germany (DLR), France (CNES), Italy (ASI), Spain (MEC), Sweden (SNSB), and the ESA Technical Directorate is gratefully acknowledged.

\section{References}

A'Hearn, M. F., Belton, M. J. S., Delamere, W. A., et al. 2011, Science, 332, 1396

Belton, M. J. S., Veverka, J., Thomas, P., et al. 1992, Science, 257, 1647

Belton, M. J. S., Chapman, C. R., Thomas, P. C., et al. 1995, Nature, 374, 785

Bertini, I., Sabolo, W., Gutierrez, P. J., et al. 2012, Planet. Space Sci., 66, 64

Boehnhardt, H. 2004, Split comets, ed. G. W. Kronk, 301

Bowell, E., Hapke, B., Domingue, D., et al. 1989, in Asteroids II, eds. R. P. Binzel, T. Gehrels, \& M. S. Matthews, 524

Chesley, S. R., Chodas, P. W., Milani, A., Valsecchi, G. B., \& Yeomans, D. K. 2002, Icarus, 159, 423

Davidsson, B., Gutiérrez, P. J., Sierks, H., et al. 2015, A\&A, 583, A16

Fornasier, S., Hasselmann, P. H., Barucci, M. A., et al. 2015, A\&A, 583, A30

Fulle, M. 1997, A\&A, 325, 1237

Fuse, T., Yoshida, F., Tholen, D., Ishiguro, M., \& Saito, J. 2008, Earth, Planets, Space, 60, 33

Hamilton, D. P., \& Burns, J. A. 1991, Icarus, 92, 118

Hermalyn, B., Farnham, T. L., Collins, S. M., et al. 2013, Icarus, 222, 625

Keller, H. U., Barbieri, C., Lamy, P., et al. 2007, Space Sci. Rev., 128, 433

Kelley, M. S., Lindler, D. J., Bodewits, D., et al. 2013, Icarus, 222, 634

Levison, H. F., \& Duncan, M. J. 1997, Icarus, 127, 13

Magrin, S., La Forgia, F., Da Deppo, V., et al. 2015, A\&A, 574, A123

Marchis, F., Boehnhardt, H., Hainaut, O. R., \& Le Mignant, D. 1999, A\&A, 349, 985

Memarsadeghi, N., McFadden, L. M., Skillman, D., et al. 2013, Proc. 2012 IS and $\mathrm{T} /$ SPIE Electronic Imaging, Computational Imaging $\mathrm{X}$ Conference

Merline, W. J., Weidenschilling, S. J., Durda, D. D., et al. 2002, Asteroids III (Tucson: University of Arizona Press), 289

Mottola, S., Lowry, S., Snodgrass, C., et al. 2014, A\&A, 569, L2

Noll, K. S., Levison, H. F., Grundy, W. M., \& Stephens, D. C. 2006, Icarus, 184, 611

Pajola, M., Vincent, J.-B., Lee, J.-C., et al. 2015, A\&A, 583, A37

Rotundi, A., Sierks, H., Della Corte, V., et al. 2015, Science, 347, 3905

Sekanina, Z. 1997, Earth Moon Planets, 77, 155

Sierks, H., Barbieri, C., Lamy, P. L., et al. 2015, Science, 347, 1044

Tubiana, C., Böhnhardt, H., Agarwal, J., et al. 2011, A\&A, 527, A113

Tubiana, C., Güttler, C., Kovacs, G., et al. 2015a, A\&A, 583, A46

Tubiana, C., Snodgrass, C., Bertini, I., et al. 2015b, A\&A, 573, A62

Veverka, J., Thomas, P., Harch, A., et al. 1999, Icarus, 140, 3

Veverka, J., Robinson, M., Thomas, P., et al. 2000, Science, 289, 2088

Weaver, H. A., \& Lamy, P. L. 1997, Earth Moon Planets, 79, 17 
1 Center of Studies and Activities for Space (CISAS) "G. Colombo", University of Padova, via Venezia 15, 35131 Padova, Italy e-mail: ivano.bertini@unipd.it

2 Instituto de Astrofísica de Andalucía - CSIC, Glorieta de la Astronomía s/n, 18008 Granada, Spain

3 Department of Physics and Astronomy "G. Galilei", University of Padova, Vicolo dell' Osservatorio 3, 35122 Padova, Italy

4 Max-Planck-Institut für Sonnensystemforschung, Justus-vonLiebig-Weg 3, 37077 Göttingen, Germany

5 Laboratoire de Astrophysique de Marseille UMR 7326, CNRS \& Aix-Marseille Université, Cedex 13, 13388 Marseille, France

${ }^{6}$ Centro de Astrobiología, CSIC-INTA, Torrejón de Ardoz, 28850 Madrid, Spain

7 International Space Science Institute, Hallerstrasse 6, 3012 Bern, Switzerland

8 Research and Scientific Support Department, European Space Agency, 2201 Noordwijk, The Netherlands

9 Department of Physics and Astronomy, Uppsala University, 75120 Uppsala, Sweden

10 PAS Space Reserch Center, Bartycka 18A, 00716 Warszawa, Polond

11 Institute for Geophysics and Extraterrestrial Physics, TU Braunschweig, 38106 Braunschweig, Germany

12 Department for Astronomy, University of Maryland, College Park, MD 20742-2421, USA

13 LESIA, Observatoire de Paris, CNRS, UPMC Univ. Paris 06, Univ. Paris-Diderot, 5 place J. Janssen, 92195 Meudon Pricipal Cedex, France
14 LATMOS, CNRS/UVSQ/IPSL, 11 boulevard d'Alembert, 78280 Guyancourt, France

15 INAF-Osservatorio Astronomico di Padova, Vicolo dell' Osservatorio 5, 35122 Padova, Italy

16 CNR-IFN UOS Padova LUXOR, via Trasea 7, 35131 Padova, Italy

17 Department of Industrial Engineering - University of Padova, via Venezia 1, 35131 Padova, Italy

18 UNITN, Universitá di Trento, via Mesiano, 77, 38100 Trento, Italy

19 Univ. Paris Diderot, Sorbonne Paris Cité, 4 rue Elsa Morante, 75205 Paris Cedex 13, France

20 INAF-Osservatorio Astronomico di Trieste, via Tiepolo 11, 34143 Trieste, Italy

21 Department of Geosciences, University of Padova, via Gradenigo 6, 35131 Padova, Italy

22 Aix-Marseille Université, CNRS, Laboratoire de Astrophysique de Marseille, UMR 7326, 13388 Marseille, France

23 Institute of Planetary Research, DLR, Rutherfordstrasse 2, 12489 Berlin, Germany

${ }^{24}$ Institute of Astronomy, National Central University, 32054 Chung-Li, Taiwan

25 Space Science Institute, Macau University of Science and Technology, Macau, PR China

${ }^{26}$ ESA/ESAC, PO Box 78, 28691 Villanueva de la Cañada, Spain

27 Institut für Datentechnik und Kommunikationsnetze, Hans-Sommer-Str. 66, 38106 Braunschweig, Germany

28 Department of Information Engineering, University of Padova, via Gradenigo 6/B, 35131 Padova, Italy

29 Physikalisches Institut, University of Bern, Sidlerstrasse 5, 3012 Bern, Switzerland 